\title{
Suppression of c-fos Induction in Rat Brain Impairs Retention of a Brightness Discrimination Reaction
}

Rita Grimm, ${ }^{1}$ Horst Schicknick, ${ }^{1}$ Isolde Riede, ${ }^{1,3}$ Eckart D. Gundelfinger, ${ }^{1}$ Thomas Herdegen, ${ }^{2}$ Werner Zuschratter, ${ }^{1}$ and Wolfgang Tischmeyer ${ }^{1,4}$

${ }^{1}$ Federal Institute for Neurobiology

D-39008 Magdeburg, Germany

${ }^{2}$ II. Institute of Physiology

University of Heidelberg

D-69120 Heidelberg, Germany

\section{Abstract}

Recently, the induction of transcription factor-encoding immediate-early genes such as c-fos was observed in distinct brain regions of rats trained to acquire a footshock-motivated brightness discrimination in a Y-maze. The functional relevance of inducible transcription factors for learning and memory formation is, however, not clear. To address this question in the present study, we have used a synthetic antisense phosphorothioate oligodeoxynucleotide to suppress in vivo the expression of c-fos in rat brain. Intrahippocampal application of the oligodeoxynucleotide $10 \mathrm{hr}$ and $2 \mathrm{hr}$ before starting a brightness discrimination training drastically reduced the induction of c-Fos immunoreactivity normally observed in limbic and cortical areas after the training session. Acquisition of the discrimination reaction was not affected by this treatment. In a relearning test $24 \mathrm{hr}$ after the first training, retention of the discrimination reaction was specifically impaired compared with rats pretreated with control oligodeoxynucleotide or saline. Our findings are consistent with the hypothesis that the inducible transcription factor c-Fos is involved in processes underlying the formation of long-term memory.

${ }^{3}$ Present address: Deutsches Krebsforschungszentrum, D-69009 Heidelberg, Germany.

${ }^{4}$ Corresponding author.

\section{Introduction}

Long-term plastic changes in the brain, including memory formation, are assumed to depend on a sequential induction of regulatory and late structural target proteins that contribute to permanent functional alterations in neuronal cells and, in particular, in their synaptic connections (Goelet et al. 1986; Alkon and Nelson 1990; Alkon et al. 1991; Armstrong and Montminy 1993). The immediateearly gene (IEG) c-fos encodes a nuclear phosphoprotein, c-Fos, which is known to be part of the AP-1 transcription factor complex (Chiu et al. 1988). The expression of this gene is rapidly and transiently induced in the central nervous system by a variety of stimuli (for recent review, see Hughes and Dragunow 1995). Moreover, numerous reports have shown increased c-fos expression after learning experiments in chicken and rodents (Anokhin et al. 1991; Anokhin and Rose 1991; Campeau et al. 1991; Bialy et al. 1992; Brennan et al. 1992; Irwin et al. 1992; Nikolaev et al. 1992a,b; Ambalavanar et al. 1993; Heurteaux et al. 1993; McCabe and Horn 1993; Pezzone et al. 1993; Houpt et al. 1994; Bertaina and Destrade 1995; Hess et al. 1995). Thus, it has been suggested that c-Fos is involved in processes of long-term memory formation, by coupling transient extracellular stimuli to long-lasting cellular responses at the level of transcription (Goelet et al. 1986; Morgan and Curran 1989).

Previous studies on rats trained to acquire a footshock-motivated brightness discrimination reaction in a Y-maze revealed a two-stage increase in hippocampal protein synthesis. Inhibition of protein synthesis during each of these phases was am-

LEARNING \& MEMORY 3:402-413 @ 1997 by Cold Spring Harbor Laboratory Press ISSN1072-0502/97 \$5.00

$$
\begin{array}{llllllllllllllll}
L & E & A & R & N & I & N & G & \begin{array}{l}
\text { V } \\
402
\end{array} & M & E & M & O & R & Y
\end{array}
$$


nesic (for review, see Matthies 1989). These findings suggested that the synthesis of proteins necessary for consolidation of the memory trace may be under the control of early induced regulatory proteins. Supporting this hypothesis, a rapid and transient induction of mRNAs encoding inducible transcription factors such as c-Fos, c-Jun, and JunB was observed in rat brain immediately after the discrimination training (Tischmeyer et al. 1990, 1994; Grimm and Tischmeyer 1997). However, similar results obtained in pseudotrained control rats suggested that initial prerequisites necessary for learning such as stimulus novelty led to an increased IEG expression during training, posing questions concerning the functional importance of IEG transcription factors for processes involved in acquisition and retention of the discrimination reaction.

Recent reports have demonstrated the usefulness of antisense (AS) oligodeoxynucleotides (ODNs) to study in vivo the role of specific proteins in the nervous system in a behavioral context (Chiasson et al. 1992; Dragunow et al. 1993; Heilig et al. 1993; Sommer et al. 1993; Merchant 1994; Möller et al. 1994; Suzuki et al. 1994; Hunter et al. 1995; Robertson et al. 1995; Wollnik et al. 1995; for review, see Wahlestedt 1994) and specifically during learning (Schmidt et al. 1995). Thus, we used intracerebral antisense intervention to assess the physiological significance of IEG transcription factors during acquisition and consolidation of the brightness discrimination in rats. Recent experiments revealed inhibitory influences of c-jun ASODN but not of $j u n B$ AS-ODN on the performance of the task (Tischmeyer et al. 1994). In the present study, we demonstrate that intrahippocampally applied c-fos AS-ODN inhibits the induction of c-Fos immunoreactivity (IR) in rat brain during brightness discrimination training and impairs the retention of the task in a sequence-specific manner.

\section{Materials and Methods}

\section{SYNTHESIS AND SEQUENCES OF ODNS}

The used ODNs, 15 nucleotides in length and phosphorothioated in all positions, were of the following sequences: c-fos AS-ODN, 5'-GAACATCATGGTCGT-3'; control ODN, 5'-TGCTGGTACTACAAG-3'. The c-fos AS-ODN was complementary to bases -6 to +9 from the initiation codon of the rat c-fos mRNA sequence. The control ODN was the reversed sequence of the c-fos AS-ODN. Neither ODN showed significant complementarity to any other gene sequence in the EMBL/GenBank databases. The ODNs were synthesized on an Applied Biosystems model 391 DNA synthesizer, purified by reverse-phase HPLC, detritylated with acetic acid, desalted, and extracted with diethyl ether. Lyophilized ODNs were resuspended to a concentration of $2 \mathrm{~mm}$ in desalted sterile water. Four independently synthesized and purified batches of each ODN were used for behavioral studies.

\section{ANIMALS AND SURGICAL PROCEDURES}

Male 7-week-old Wistar rats were deeply anesthetized with pentobarbital ( $60 \mathrm{mg} / \mathrm{kg}$ i.p.) and stereotaxically implanted with stainless-steel microcannulae bilaterally into the dorsal hippocampus using the following coordinates: posterior -2.8 $\mathrm{mm}$ from bregma, lateral $\pm 2.2 \mathrm{~mm}$, and ventral $-3.2 \mathrm{~mm}$ from skull. According to the brain atlas from Paxinos and Watson (1986), these coordinates correspond to the CA1 region in the dorsal hippocampus. Guide cannulae were fixed using anchor screws and acrylic cement. Following surgery, rats were housed singly and given free access to standard laboratory chow and tap water on a 12-hr light/dark cycle (light on at 6 a.m.). During a 1-week recovery period before beginning the experiments, rats were handled daily to habituate to the injection procedure.

\section{INTRAHIPPOCAMPAL INJECTIONS}

For administration of saline or ODNs to freely moving rats, blunt-tipped stainless-steel injectors were placed gently into the guide cannulae such that the tips were located $0.1 \mathrm{~mm}$ below the cannulae. For behavioral studies, $1 \mu \mathrm{l}$ of $0.9 \%$ saline or of a $2 \mathrm{~mm}$ solution of c-fos AS-ODN or control ODN was injected twice into each hippocampus at a rate of $\sim 0.5 \mu \mathrm{l} / \mathrm{min}$. Subsequently, injectors were left in place for another minute. The first injection was given $10 \mathrm{hr}$ prior to training, considering previous reports of an $\sim 8$-hr onset latency for c-fos AS-ODN (Chiasson et al. 1992; Suzuki et al. 1994). To maintain efficient local concentrations of ODNs during acquisition and subsequent phases of consolidation of the discrimination reaction, a second injec-

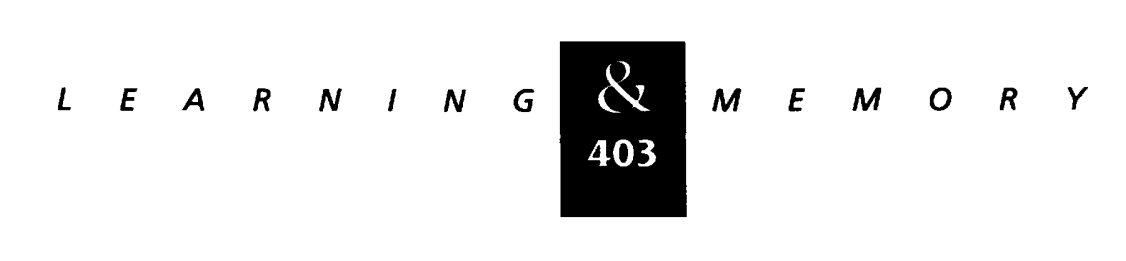


tion was given $2 \mathrm{hr}$ before the beginning of training. A significant suppression of c-Fos IR within a period of $3 \mathrm{hr}$ following intracerebral administration of c-fos AS-ODN has been demonstrated recently (Shih et al. 1996).

To immunohistochemically examine the effects of c-fos AS-ODN on c-fos expression, six rats were injected with c-fos AS-ODN into one hippocampus and control ODN into the opposite one using the same protocol as described above. The use of the right and left hemispheres for injecting the different ODNs was changed between animals.

\section{IMMUNOHISTOCHEMISTRY}

\section{TRAINING-INDUCED EXPRESSION OF C-FOS IR}

To examine the induction of Fos protein in rat brain after brightness discrimination training, rats $(n=4)$ without surgical or pharmacological pretreatments were trained in a Y-maze as described below. Another group of rats $(n=4)$ was allowed to stay in the home cage during the time of training to serve as passive controls. Immediately after completion of one session of training, rats were deeply anesthetized with sodium pentobarbital. Following perfusion fixation first with $0.1 \mathrm{M}$ phosphate-buffered saline (PBS) (pH 7.4) and then with 4\% paraformaldehyde dissolved in the same buffer, brains were removed and sectioned on a vibratome. Immunohistochemistry was performed on free-floating $50-\mu \mathrm{m}$ coronal sections using affinitypurified polyclonal c-Fos antibodies (Santa Cruz Biotechnology, Inc.), raised in rabbits by immunization with a peptide corresponding to human cFos amino acid residues 3-16. As tested by immunoprecipitation, Western blotting, and cell staining (Sambucetti and Curran 1986), these antibodies react with vertebrate c-fos-encoded $62-\mathrm{kD}$ proteins. To reduce background staining, sections were preincubated in a mixture of $50 \%$ methanol/PBS with $0.1 \%$ hydrogen peroxide for $15 \mathrm{~min}$ to block endogeneous peroxidases and in $5 \%$ bovine serum albumin for $1 \mathrm{hr}$ to block unspecific binding. Subsequently, sections were incubated in the primary antisera $(1: 20,000)$, including $0.1 \%$ Triton X-100 for $36 \mathrm{hr}$. After thorough washing in PBS, sections were incubated with anti-rabbit-digoxigenin (1:100; Boehringer Mannheim) followed by antidigoxigenin-peroxidase, Fab fragments (1:400; Boehringer Mannheim). Visualization of labeled cells was performed by developing sections in $0.02 \%$ diaminobenzidine with $0.02 \%$ hydrogen peroxide.

SUPPRESSION OF C-FOS IR EXPRESSION BY C-fos AS-ODN

To determine the efficacy of c-fos AS-ODN, rats $(n=6)$ were twice injected in opposite hippocampi with c-fos AS-ODN and control ODN as described above and trained on a brightness discrimination reaction (see below) $2 \mathrm{hr}$ after the second application of ODNs. Immmediately after completion of one session of training, rats were deeply anesthetized by sodium pentobarbital and perfused transcardially with $4 \%$ paraformaldehyde. Brains were removed, postfixed in the same fixative overnight, and cryoprotected in $30 \%$ sucrose. Coronal sections $(50 \mu \mathrm{m})$ were prepared on a cryostat and processed for immunohistochemistry. Primary antiserum against c-Fos (Kovary and Bravo 1991) was diluted 1:12,000. Detection was performed as described previously using an avidin-biotin protocol (Herdegen et al. 1991). Briefly, sections were incubated with primary antiserum for $48-72 \mathrm{hr}$ at $4^{\circ} \mathrm{C}$, washed, and reincubated with secondary antibody (polyclonal anti-rabbit antiserum) for $45 \mathrm{~min}$ at $37^{\circ} \mathrm{C}$, washed again, and processed with an $\mathrm{ABC}$ Elite kit (Vectastain, Vector Laboratories, Inc.). As described previously (Herdegen et al. 1991; Kovary and Bravo 1991), the rabbit anti c-Fos antibody shows a strong reaction with the c-Fos protein, no cross-reactivity with FosB and a weak reaction with the Fos-related antigen Fra-1. For quantitative analysis, immunopositive cells in frames of 1 $\mathrm{mm} \times 1 \mathrm{~mm}$ covering the apex of the dentate gyrus were counted separately on control ODN-treated and c-fos AS-ODN-treated hemispheres. Values obtained from two slices per rat were averaged for statistical evaluation.

\section{BRIGHTNESS DISCRIMINATION EXPERIMENTS}

\section{HABITUATION}

Brightness discrimination training and a relearning test $24 \mathrm{hr}$ after the initial training were performed in a Y-shaped chamber. Before both the training and the relearning session, rats were allowed to habituate for $10 \mathrm{~min}$ to the experimental situation in the Y-maze without illumination and

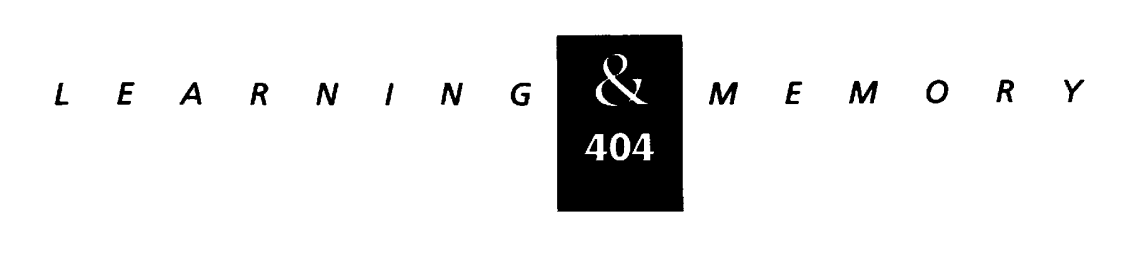


footshock. To assess effects of ODNs on arousal and activity, the number of entries into each arm of the Y-maze was monitored during this period.

TRAINING

The animals were trained in the Y-maze to attain a footshock-motivated brightness discrimination, as described earlier (Tischmeyer et al. 1994). Briefly, the learning task was to escape from a mild electrical footshock (1-1.5 mA) from the grid floor in the start box by running into the illuminated alley of the Y-maze. At the beginning of each trial, the goal box was illuminated and a foot shock was delivered to the start box. When the animal escaped from the footshock into the illuminated goal box, the shock was terminated and the light remained on for another $20 \mathrm{sec}$. Entering the dark alley was punished by footshock and counted as an error. Rats stayed in the goal box for a randomly changing intertrial interval of $\sim 1 \mathrm{~min}$. For the next trial, the former goal box served as the start box, thus avoiding intertrial handling of the animals. The side of illumination was changed after three consecutive runs. A training session consisted of 40 trials and was completed within $45 \mathrm{~min}$. To assess effects of ODNs on motor coordination and sensory systems, the following parameters were recorded during each run: (1) footshock intensity required to cause the rats to escape from the start box, (2) reaction time, that is, the time of escape from the start box after the onset of footshock, and (3) running time, that is, the time required to escape into the goal box.

\section{RELEARNING}

Retention was estimated in a relearning test 24 $\mathrm{hr}$ after the initial training using the same test apparatus and the same behavioral procedure. Parameters were recorded as described above. The reduction in the number of errors compared with the initial training was expressed as an index of retention (\% savings), calculated as

$$
\begin{aligned}
& \text { \% savings }= \\
& \frac{\text { training errors - relearning errors }}{\text { training errors }}
\end{aligned}
$$

Following the behavioral experiments, the correct positions of cannulae tips were determined.
Briefly, animals were sacrificed by decapitation; brains were removed from the skull and fixed by immersion with $4 \%$ paraformaldehyde for 5 days. Localization of the cannulae was checked on 50 $\mu \mathrm{m}$ vibratome sections. Only animals that had the cannulae inserted into the appropriate location were chosen for further analysis of the data.

\section{STATISTICAL ANALYSIS}

All values are expressed as means \pm S.E.M. OWing to the small sample size, immunohistochemical data were statistically analyzed using Wilcoxon's signed rank test. Statistical evaluation of behavioral data was performed using one-way ANOVA. If significant, post-hoc comparisons were performed using Student's two-tailed $t$-test. $P$ values of $<0.05$ were considered as statistically significant.

\section{Results}

\section{TRAINING-INDUCED EXPRESSION OF C-FOS IR}

Immediately after completion of a 45-min training session in the $\mathrm{Y}$-maze, a distinct expression pattern of c-Fos IR was observed in several rat brain regions such as the hippocampal formation, the parietal, perirhinal, and piriform cortices, the amygdala, and thalamic nuclei, whereas in passive control rats, only a few scattered cells were immunopositive. In Figure 1, the hippocampal CA1 region, the dentate gyrus, and the parietal cortex (area 1) are shown as examples. Training-induced expression of c-Fos IR was observed in pyramidal cells of the hippocampal region CA1 (and CA3, not shown), in granule cells of the dentate gyrus, and in hilar cells. However, in the CA2 region, c-Fos IR remained undetectable after training (not shown). In the parietal cortex, c-Fos IR was predominantly induced in layers IV and VI after the discrimination training (Fig. 1). In all regions, localization of c-Fos IR was apparently restricted to the nuclei of labeled cells.

\section{SUPPRESSION OF C-FOS IR EXPRESSION BY c-fos AS-ODN}

To compare the effects of c-fos AS-ODN versus control ODN on the expression of c-Fos IR, a group of six rats received c-fos AS-ODN into one hippocampus, whereas control ODN was applied to the contralateral hippocampus of the same animal. Us-

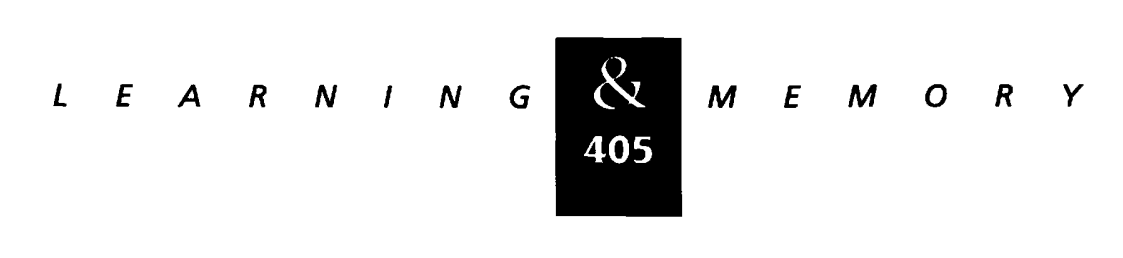


passive control

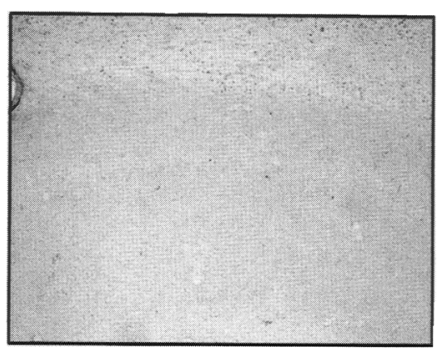

Figure 1: Training-induced expression of C-Fos IR in rat brain. Rats $(n=4$ per group) without surgical or pharmacological pretreatments were either trained on a brightness discrimination in a Y-maze or stayed in their home cage during the time of training to serve as passive controls. Immediately after completion of training, rats were anesthetized and perfused. Brains were removed and processed for immunohistochemistry using the digoxigenin protocol as described in Materials and Methods. Details of coronal sections at the level of the hippocampal CA1 region (CA1), the dentate gyrus (DG), and the area 1 of the parietal cortex (Ctx) of a trained rat (right) and a passive control (left) are shown as representative examples.
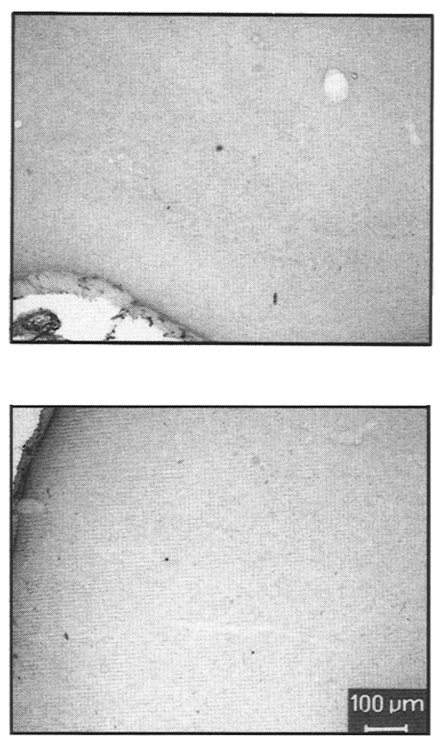

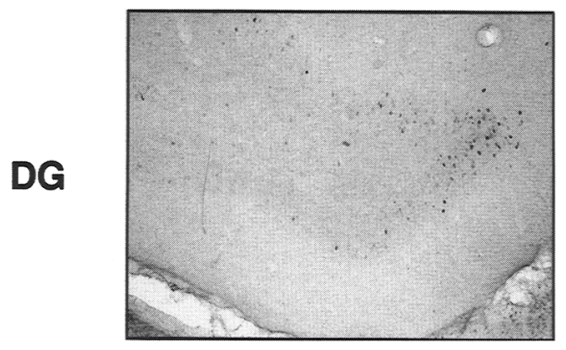

immediately after training

CA1
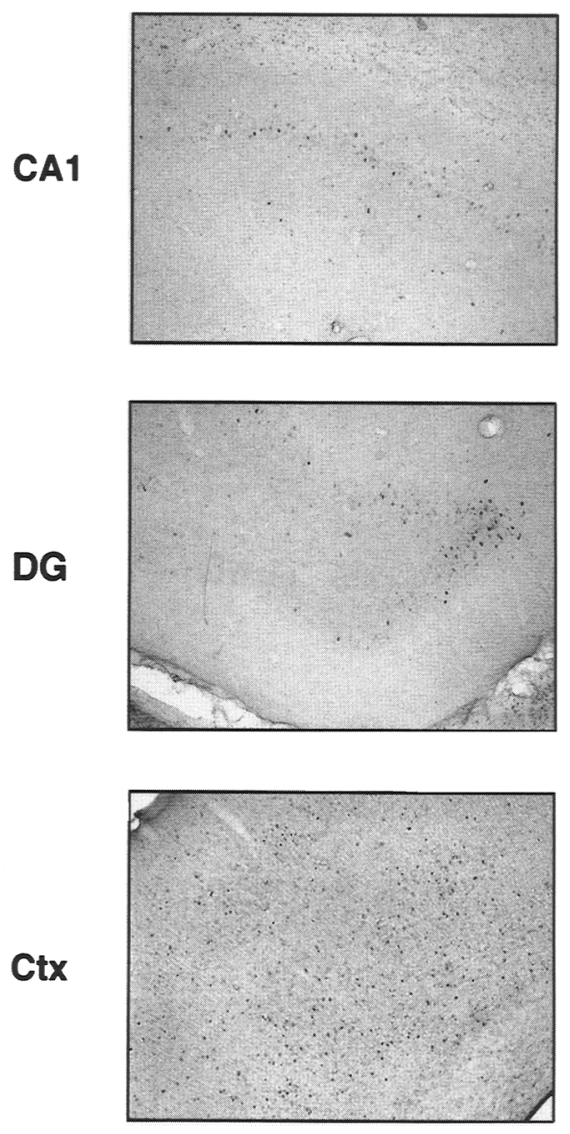

ing the more sensitive avidin-biotin protocol, a strong expression of c-Fos IR was detected in several brain regions of the control ODN-injected hemisphere following brightness discrimination training (Fig. 2), including principal neurons of the hippocampal formation (pyramidal neurons of the cornus ammonis, granular cells of the dentate gyrus) and cells of the overlying cortex. In contrast, on the c-fos AS-ODN-injected hemisphere, strikingly less c-Fos IR was observed in large areas of the hippocampus, the dentate gyrus, and the adjacent cortex. To assess the efficacy of c-fos AS-ODN, immunopositive cells were counted in areas of 1 $\mathrm{mm}^{2}$ covering the apex of the dentate gyrus. On average, the number of stained cells was reduced from $355 \pm 151$ on control ODN-treated hemispheres to $56 \pm 13$ on c-fos AS-ODN-treated hemispheres ( $n=6, P<0.05$, Wilcoxon test), representing $>70 \%$ inhibition. The action of c-fos ASODN was highly restricted to the ipsilateral hemisphere. However, a few scattered cells, for example, in the hilar region, still expressed c-Fos
IR also in this hemisphere (Fig. 2). Moreover, whereas in four out of six rats the suppression of c-Fos IR by c-fos AS-ODN was comparable in extent to the example shown in Figure 2, less reduction was observed in the remaining two rats (data not shown).

\section{BRIGHTNESS DISCRIMINATION EXPERIMENTS}

TRAINING

Pretraining injection of c-fos AS-ODN or control ODN caused no alterations in habituation compared with saline-treated rats. As shown in Table 1, the number of runs during $10 \mathrm{~min}$ of habituation before the initial training was comparable in salinetreated, control ODN-treated and c-fos AS-ODNtreated rats. Furthermore, as revealed by ANOVA, parameters recorded during the training session such as footshock intensities $[F(2,40)=0.39$, $P>0.10]$, reaction times $[F(2,40)=0.70, P>0.10]$,

$$
\ldots \ldots, n \text { d }
$$


A

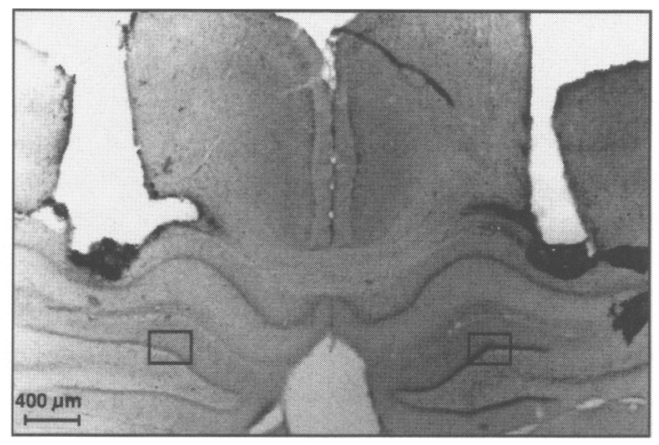

B

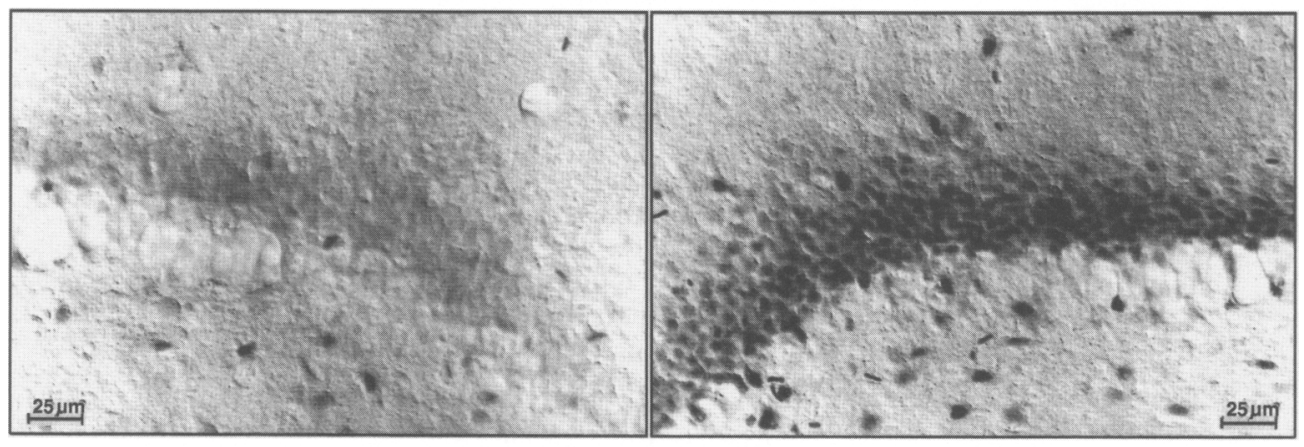

c- fos AS-ODN

control-ODN

Figure 2: Suppression of c-Fos IR expression by c-fos AS-ODN in hippocampal and cortical regions of rat brain. Ten hours and $2 \mathrm{hr}$ before the beginning of a brightness discrimination training, the rat received c-fos AS-ODN to the right and control ODN to the left hemisphere of the brain. Immediately after completion of training, the animal was anesthetized and perfused. The brain was removed and processed for immunohistochemistry using the avidin-biotin protocol as described in Materials and Methods. (A) Detail of a coronal section (frontal view) showing hippocampal and cortical regions surrounding the injection sites. $(B)$ Magnifications of the dentate granule cell layers boxed in $A$.

and running times $[F(2,40)=1.27, P>0.10]$ did not significantly differ among groups.

Compared with saline-treated and control ODN-treated rats, performance of brightness discrimination training was not impaired in c-fos ASODN-treated animals, as documented by comparable numbers of training errors in all three groups (Table 1).

\section{RELEARNING}

During the habituation period before the relearning session, the number of runs between the arms of the Y-maze performed by saline-treated as well as control ODN-treated rats was significantly reduced compared with the exploratory behavior of these rats before the initial training (Table 1). In contrast, the number of runs performed by c-fos AS-ODN-treated rats during the habituation period before the relearning test was almost identical to the pretraining habituation performance observed in this group.

As for the initial training, ANOVA revealed no significant group differences in general parameters, such as footshock intensities $[F(2,40)=0.15$, $P>0.10], \quad$ reaction times $[F(2,40)=1.69$, $P>0.10]$, and running times $[F(2,40)=3.02$, $P>0.05]$, recorded during the relearning test. However, compared with saline-treated rats, running timestended to increase in rats treated with control ODN or c-fos AS-ODN (Table 1). To assess interactions between running times and the brightnessdiscrimination score, linear regression analysis wasperformed. It revealed no significant correlationbetween running times and the number of relearning errors $[r=0.25, F(1,41)=2.78, P>0.10]$.

Compared with the initial training, the number of errors during the relearning session was significantly reduced in saline-treated, in control ODNtreated as well as in c-fos AS-ODN-treated rats (Table 1), indicating an improvement of the bright-

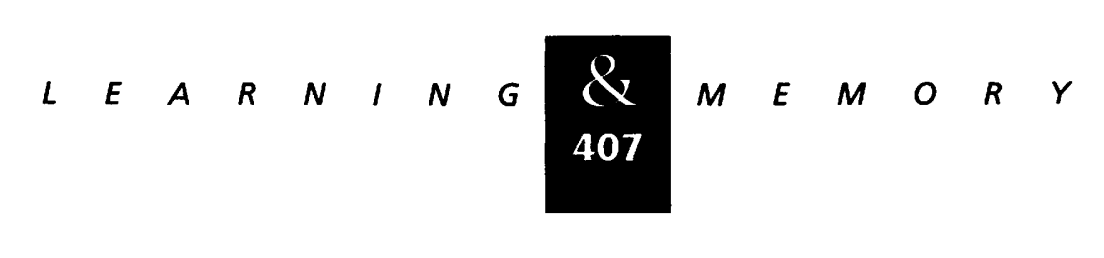


Grimm et al.

Table 1: Data recorded during 10 min of habituation to the $Y$-maze, during brightness discrimination training, and during the relearning test $24 \mathrm{hr}$ after the initial training

\begin{tabular}{|c|c|c|c|c|c|c|}
\hline & \multicolumn{3}{|c|}{ Training } & \multicolumn{3}{|c|}{ Relearning } \\
\hline & $\begin{array}{c}\text { saline } \\
(n=12)\end{array}$ & $\begin{array}{c}\text { control } \\
\text { ODN } \\
(n=16)\end{array}$ & $\begin{array}{c}\text { c-fos AS-ODN } \\
(n=15)\end{array}$ & $\begin{array}{c}\text { saline } \\
(n=12)\end{array}$ & $\begin{array}{c}\text { control } \\
\text { ODN } \\
(n=16)\end{array}$ & $\begin{array}{c}\text { c-fos AS-ODN } \\
(n=15)\end{array}$ \\
\hline $\begin{array}{l}\text { Runs during } \\
\text { habituation }\end{array}$ & $24.7 \pm 1.5$ & $26.9 \pm 1.7$ & $21.7 \pm 1.9$ & $9.0 \pm 2.8^{\mathrm{a}}$ & $11.6 \pm 1.8^{\mathrm{a}}$ & $19.7 \pm 3.2^{b}$ \\
\hline $\begin{array}{l}\text { Shock intensities } \\
\quad(\mathrm{mA}) \\
\text { Reaction times }\end{array}$ & $1.28 \pm 0.01$ & $1.30 \pm 0.02$ & $1.27 \pm 0.02$ & $1.29 \pm 0.01$ & $1.28 \pm 0.03$ & $1.30 \pm 0.01$ \\
\hline $\begin{array}{l}\text { (sec) } \\
\text { Running times }\end{array}$ & $1.66 \pm 0.26$ & $1.74 \pm 0.28$ & $2.19 \pm 0.43$ & $1.29 \pm 0.25$ & $2.24 \pm 0.36$ & $2.19 \pm 0.48$ \\
\hline (sec) & $3.60 \pm 0.40$ & $4.51 \pm 0.39$ & $4.71 \pm 0.63$ & $2.25 \pm 0.27^{\mathrm{a}}$ & $3.67 \pm 0.37^{\mathrm{a}}$ & $3.92 \pm 0.68$ \\
\hline $\begin{array}{l}\text { errors } \\
\text { eromber of }\end{array}$ & $13.4 \pm 1.0$ & $14.3 \pm 0.6$ & $13.2 \pm 0.7$ & $6.5 \pm 1.0^{\mathrm{a}}$ & $7.0 \pm 0.8^{\mathrm{a}}$ & $9.3 \pm 1.2^{\mathrm{a}}$ \\
\hline
\end{tabular}

Rats were exposed to the $Y$-maze for $10 \mathrm{~min}$ before both the training and the relearning session to equilibrate to the experimental environment. During these habituation periods, runs between arms of the Y-maze were recorded. Footshock intensities, reaction times, running times, and the number of errors were monitored during the subsequent brightness discrimination training, and during the relearning test $24 \mathrm{hr}$ later. Means \pm S.E.M.. Number of animals are in parenthesis. ${ }^{a}(P<0.05)$ Significantly different from the respective values obtained on the training day of the same group of rats (paired $t$-test).

${ }^{b}(P<0.05)$ Significantly different from the respective values of saline-treated and control ODN-treated rats on the day of relearning (unpaired $t$-test).

ness discrimination score in all three groups. However, in comparison with rats pretreated with saline or control ODN, this reduction in the number of relearning errors was less pronounced in the group of c-fos AS-ODN-injected rats, implying a deficit in retention. Calculation of the retention index supported these findings. As shown in Figure 3, percent savings were similar in saline-treated and control ODN-treated rats, whereas in c-fos ASODN-treated rats, the retention index was significantly reduced.

\section{Discussion}

Using an antisense approach, we addressed the question of the functional relevance of inducible transcription factors for processes of learning and memory formation. As demonstrated in the present study, the expression of c-Fos IR is induced in various rat brain regions following training on a footshock-motivated brightness discrimination reaction in a Y-maze. Intracerebral injection of c-fos AS-ODN prior to training efficiently suppresses cFos expression in hippocampal and cortical areas. This has amnesic effects in a relearning test $24 \mathrm{hr}$ later, whereas the acquisition of the new behavior is not affected.

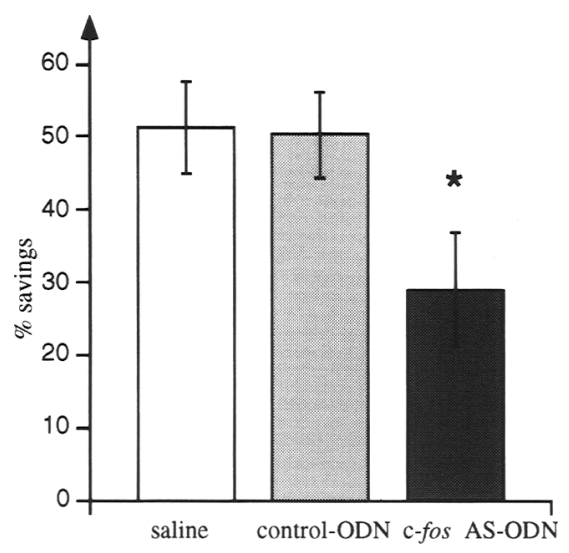

Figure 3: Retention indices expressed as percent savings. The retention indices were calculated from the number of training errors and relearning errors of salinetreated $(n=12)$, control ODN-treated $(n=16)$ and c-fos AS-ODN-treated ( $n=15)$ rats as described in Materials and Methods. Means \pm S.E.M. $\left(^{*}\right) P<0.05$ : significantly different from the values of saline-treated and control ODN-treated rats (unpaired $t$-test).

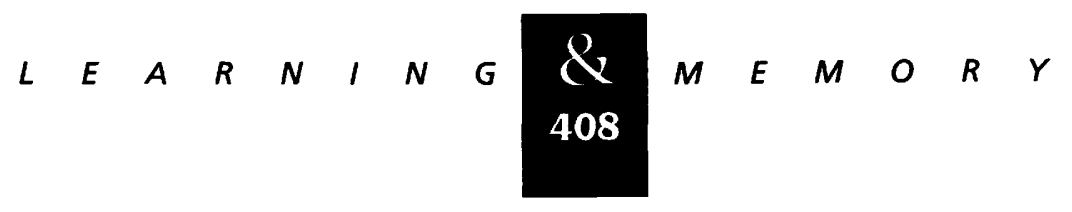


Hybridization studies have previously revealed a differential induction of transcription factorencoding IEGs including c-fos in hippocampal, cortical, and cerebellar rat brain structures following brightness discrimination training (Tischmeyer et al. 1990, 1994; Grimm and Tischmeyer 1997). These findings at the level of mRNA implied but did not prove an increased expression also of the respective proteins. Using immunohistochemical methods, we therefore examined the induction of c-fos gene expression at the translational level. As shown in Figure 1, c-Fos IR was induced in various rat brain regions including limbic and cortical structures immediately after completion of the Y-maze training, confirming reports on an increased expression of Fos protein in chick, mouse, rat, and rabbit brain following different behavioral paradigms (Brennan et al. 1992; Irwin et al. 1992; Ambalavanar et al. 1993; McCabe and Horn 1993; Pezzone et al. 1993; Freeman and Rose 1995). Our findings demonstrate that the induction of c-fos gene expression observed at the level of mRNA is continued at the translational level to increase the amount of c-Fos protein during acquisition and early phases of consolidation of the discrimination reaction, suggesting a physiological involvement of this transcription regulatory protein in the formation of the memory trace.

AS-ODNs are commonly designed to inhibit the synthesis of specific proteins by hybridization to the respective mRNA. One of the major problems for in vivo use of these compounds is their rapid degradation by nucleolytic enzymes. More stable ODNs can be generated by replacing a nonbridging oxygen of the phosphodiester backbone by sulfur at each base. The resulting phosphorothioated ODNs are known to be effectively taken up by brain cells and to be stable there for $>24 \mathrm{hr}$ after topical application in vivo (Whitesell et al. 1993; Wahlestedt 1994). Using intrahippocampal injection in rats, we have demonstrated earlier that labeled phosphorothioated ODNs were detectable in hippocampal pyramidal cells for at least $26 \mathrm{hr}$ and, as shown for c-jun AS-ODN, behavioral effects were also still evident at this time (Tischmeyer et al. 1994). On the other hand, unmodified c-fos ASODN intrahippocampally applied at the same dosage as the phosphorothioated one was ineffective in the present study (data not shown), confirming previous observations in rat brain after intrastriatal injections (Hooper et al. 1994). Thus, to examine the role of Fos protein in rat brain during learning and memory formation, we used phosphorothioated ODNs.

The c-fos AS-ODN used in our experiments has been shown previously to successfully inhibit c-Fos expression in rat brain and spinal cord following topical application (Chiasson et al. 1992; Dragunow et al. 1993; Heilig et al. 1993; Sommer et al. 1993; Hooper et al. 1994; Merchant 1994; Möller et al. 1994; Hunter et al. 1995; Quercia and Chang 1996; Shih et al. 1996). Similarly, using an intrahippocampal application protocol in the present study, we observed an efficient suppression of cFos IR in hippocampal and cortical regions ipsilateral to the injection site of c-fos AS-ODN compared with the contralateral, control ODN-injected hemisphere of the same rat after brightness discrimination training (Fig. 2). However, single scattered cells of the ipsilateral hemisphere, for example, in the hilar region, still expressed c-Fos IR, probably owing to cell type-specific differences in the uptake of the ODN (Sommer et al. 1993). Furthermore, inhibition of c-fos induction by c-fos AS-ODN is not always complete. Thus, in four out of six rats a strong suppression of c-Fos IR was evident, whereas in two rats the inhibition was clearly weaker. The reasons for this latter finding are not known.

Intracerebrally applied phosphorothioated ODNs may show toxic effects, including tissue damage (Chiasson et al. 1994). However, neither behavioral effects nor signs of structural damage were observed when amounts of c-fos AS-ODN comparable to those used in the present study were applied to the amygdala several days (i.e., with an interval that allows degradation of ODNs) before training or shortly before testing of rats in a conditioned taste aversion paradigm (Lamprecht and Dudai 1996). Similarly, in the present study no tissue damage additional to injuries caused by the insertion of cannulae was observed in ODN-treated versus saline-treated rats during determination of cannulae positions 5 days following intrahippocampal injections (data not shown). Nevertheless, to control for sequence-independent effects of cfos AS-ODN, behavioral experiments were performed in comparison with saline-treated as well as control ODN-treated rats. The control ODN used was a reversed AS-ODN; that is, the nucleotide sequence was assembled in inverted orientation to prevent hybridization to c-fos mRNA and gene. Compared with commonly used control ODNs, such as sense, mismatched, or scrambled ODNs, reversed AS-ODNs advantageously have identical

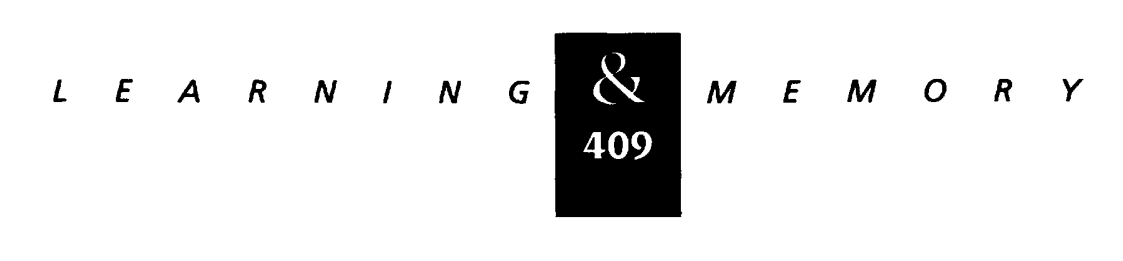


nucleotide compositions and structural features as the corresponding AS-ODNs. Furthermore, physiological effects occasionally observed with sense (Cameron and Jennings 1991; Scherczinger et al. 1992) and mismatched (Woolf et al. 1992; Chiasson et al. 1994; Tischmeyer et al. 1994) sequences should not occur with reversed AS-ODNs.

Recently, severe behavioral deficits have been described in mutant mice lacking c-fos expression (Paylor et al. 1994). However, these mice showed impaired sensitivities of sensory systems and had heterogeneous genetic backgrounds, making interpretation of learning deficits rather difficult. Hence, to look for side effects of the administered ODNs per se and of the suppression of c-fos expression on mechanisms that may interfere with the learning performance, we have analyzed whether ODN-induced alterations occur during adaptation of the rats to the test apparatus. Runs between the arms of the Ymaze were counted during the habituation period before both the training and the relearning test and assessed as an index of arousal and activity. On the day of training, habituation performances of control ODN-treated rats as well as of c-fos AS-ODN-treated animals did not differ from exploratory behavior of the saline-treated control group (Table 1), indicating that neither control ODN nor c-fos AS-ODN caused alterations in the state of arousal and activity. These findings are in accordance with previous results demonstrating that intrahippocampally applied phosphorothioated ODNs did not alter the activity of rats in an open field test (Tischmeyer et al. 1994). On the relearning day, again saline-treated and control ODN-treated rats showed almost identical habituation performances, but the number of runs performed by these rats was reduced significantly compared with their pretraining exploratory activity, probably reflecting some kind of familiarity with the training chamber and the experimental situation. In contrast, exploratory behavior of c-fos AS-ODN-treated rats during the habituation phase on the relearning day did not significantly differ from that on the day of initial training when the test apparatus was novel to these rats, suggesting that they did not remember the experimental situation well and therefore behaved like naive animals.

During training and relearning of the brightness discrimination reaction, general parameters such as footshock intensities as well as reaction times and running times required to escape from the start box and to arrive in the goal box, respectively, were recorded. These parameters reflect the sensitivity of sensory and motor systems. They did not vary significantly among the differently pretreated groups of rats. A tendency to increased running times was noticeable both in control ODNtreated and c-fos AS-ODN-treated rats during relearning. This trend, however, did not correlate with the brightness discrimination score. Thus, neither the ODNs per se nor the suppression of c-fos expression in c-fos AS-ODN-treated rats caused deficits in sensory transduction that could account for learning deficits.

In aggreement with the observed normal motor and sensory behavior, the number of training errors did not differ between groups (Table 1). This shows that acquisition of the brightness discrimination reaction was not affected by the two ODNs. In contrast, after $24 \mathrm{hr}$, the degree of retention of the task was clearly diminished in c-fos AS-ODN-treated rats, as reflected in a significantly reduced retention index compared with the level of both the saline-treated and the control ODN-treated groups (Fig. 3). However, pretraining application of c-fos AS-ODN did not completely prevent brightness discrimination learning, probably reflecting an incomplete inhibition of cfos expression or interindividual variations in the application, penetration, or efficiency of c-fos ASODN as could be expected from the immunohistochemical data. Alternatively, related transcription factors might compensate to a certain degree for C-Fos.

Recently, we have shown that suppression of c-Jun expression by intrahippocampally applied AS-ODN affected mainly the acquisition of brightness discrimination in rats (Tischmeyer et al. 1994), implying that this transcription regulatory protein, expressed at a relatively high basal level in rodent brain, is necessary for normal neuronal function. In contrast, suppression of another member of the Jun family, JunB, was without effects on the discrimination performance. Our present results demonstrate a sequence-specific impairment of retention of the brightness discrimination reaction in rats by intrahippocampally applied c-fos ASODN. The parameters monitored during the behavioral experiments indicate that this retention deficit does not result from influences on sensory or motor systems that may interfere with the acquisition of the discrimination task, suggesting that $c$ Fos, certainly in combination with other transcription factors, is involved in mechanisms of consoli-

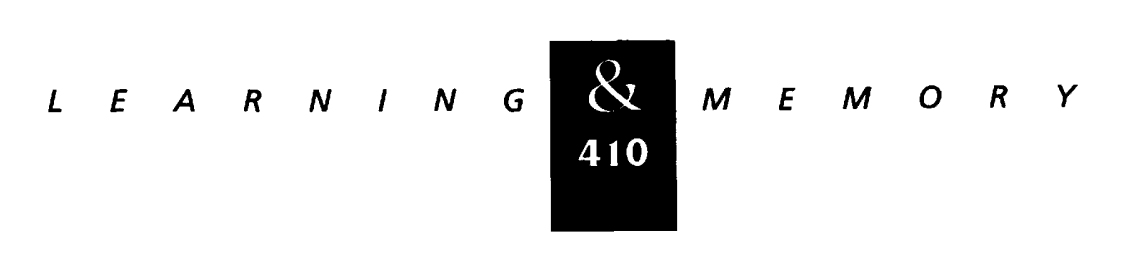


dation and/or retrieval of the discrimination reaction.

Furthermore, as published during the reviewing of this manuscript, transient expression of cFos in rat amygdala is required for encoding conditioned taste aversion memory (Lamprecht and Dudai 1996). Thus, results yielded by use of different learning paradigms support the hypothesis that c-Fos is a link in the chain of metabolic events induced by neuronal signals during learning and leading finally to the formation of a long-term memory trace.

\section{Acknowledgments}

We thank K. Schuhmacher, G. Domnick, and A. Bolz for excellent technical assistance. This work was supported by the Ministerium für Wissenschaft und Forschung, Land Sachsen-Anhalt, grants 074A1021 and 074A10213.

The publication costs of this article were defrayed in part by payment of page charges. This article must therefore be hereby marked "advertisement" in accordance with 18 USC section 1734 solely to indicate this fact.

\section{References}

Alkon, D.L. and T.J. Nelson. 1990. Specificity of molecular changes in neurons involved in memory storage. FASEB J. 4: 1567-1576.

Alkon, D.L., D.G. Amaral, M.F. Bear, J. Black, T.J. Carew, N.J. Cohen, J.F. Disterhoft, H. Eichenbaum, S. Golski, L.K. Gorman, G. Lynch, B.L. McNaughton, M. Mishkin, J.R. Moyer Jr., J.L. Olds, D.S. Olton, T. Otto, L.R. Squire, L.R. Staubli, L.T. Thompson, and C. Wible. 1991. Learning and memory. Brain Res. Rev. 16: 193-220.

Ambalavanar, R., E.A. Vanderzee, J.J. Boehnis, B.J. McCabe, and J. Horn. 1993. Coexpression of FOS immunoreactivity in protein kinase gamma-positive neurons, quantitative analysis of a brain region involved in learning. Brain Res.

606: $315-318$.

Anokhin, K.V. and S.P.R. Rose. 1991. Learning-induced increase of immediate early gene messenger RNA in the chick forebrain. Eur. J. Neurosci. 3: 162-167.

Anokhin, K.V., R. Mileusnic, I.Y. Shamakina, and S.P.R. Rose. 1991. Effects of early experience on c-fos gene expression in the chick forebrain. Brain Res. 544: 101-107.

Armstrong, R.C. and M.R. Montminy. 1993. Transsynaptic control of gene expression. Annu. Rev. Neurosci. 16: 17-29.

Bertaina, V. and C. Destrade. 1995. Differential time course of c-fos mRNA expression in hippocampal subfields following acquisition and recall testing in mice. Cognit. Brain Res. 2: 269-275.

Bialy, M., E. Nikolaev, J. Beck, and L. Kaczmarek. 1992.
Delayed c-fos expression in sensory cortex following sexual learning in male rats. Mol. Brain Res. 14: 352-356.

Brennan, P.A., D. Hancock, and E.B. Keverne. 1992. The expression of the immediate-early genes c-fos, egr-1 and c-jun in the accessory olfactory bulb during the formation of an olfactory memory in mice. Neuroscience 4: 277-284.

Cameron, F.N. and P.A. Jennings. 1991. Inhibition of gene expression by a short sense fragment. Nucleic Acids Res. 19: 469-475.

Campeau, S., M.D. Hayward, B.T. Hope, J.B. Rosen, E.J. Nestler, and M. Davis. 1991. Induction of the c-fos proto-oncogene in rat amygdala during unconditioned and conditioned fear. Brain Res. 565: 349-352.

Chiasson, B.J., M.L. Hooper, P.R. Murphy, and H.A. Robertson. 1992. Antisense oligonucleotide eliminates in vivo expression of c-fos in mammalian brain. Eur. J. Pharmacol. 227: 451-452.

Chiasson, B.J., J.N. Armstrong, M.L. Hooper, P.R. Murphy, and H.A. Robertson. 1994. The application of antisense oligonucleotide technology to the brain: Some pitfalls. Cell. Mol. Neurobiol. 14: 507-521.

Chiu, R., W.J. Boyle, J. Meek, T. Smeal, T. Hunter, and M. Karin. 1988. The c-fos protein interacts with c-jun /AP1 to stimulate transcription of AP-1 responsive genes. Cell 54: 541-552.

Dragunow, M., P. Lawlor, B. Chiasson, and H.A. Robertson. 1993. c-fos antisense generates apomorphine and amphetamine-induced rotation. NeuroReport 5: 305-306.

Freeman, F.M. and S.P.R. Rose. 1995. MK-801 blockade of Fos and Jun expression following passive avoidance training in the chick. Eur. J. Neurosci. 7: 563-569.

Goelet, P., V.F. Castellucci, S. Schacher, and E.R. Kandel. 1986. The long and the short of long-term memory-a molecular framework. Nature 322: 419-423.

Grimm, R. and W. Tischmeyer. 1997. Complex patterns of immediate early gene induction in rat brain following brightness discrimination training and pseudotraining. Behav. Brain Res. 84: 109-116.

Heilig, M., J.A. Engel, and B. Söderpalm. 1993. c-fos antisense in the nucleus accumbens blocks the locomotor stimulant action of cocaine. Eur. J. Pharmacol. 236: 339-340.

Herdegen, T., K. Kovary, J. Leah, and R. Bravo. 1991. Specific temporal and spatial distribution of Jun, Fos and Krox-24 proteins in spinal neurons following noxious transsynaptic stimulation. J. Comp. Neurol. 312: 1-14.

Hess, U.S., G. Lynch, and C.M. Gall. 1995. Changes in c-fos mRNA expression in rat brain during odor discrimination learning: Differential involvement of hippocampal subfields CA1 and CA3. J. Neurosci. 15: 4786-4795.

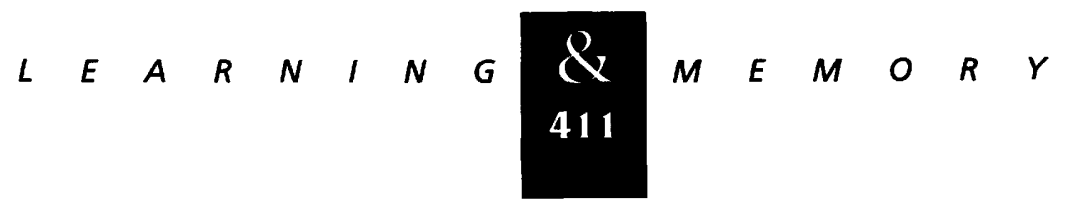


Heurteaux, C., C. Messier, C. Destrade, and M. Lazdunski. 1993. Memory processing and apamin induced immediate early gene expression in mouse brain. Mol. Brain Res. 3: $17-22$.

Hooper, M.L., B.J. Chiasson, and H.A. Robertson. 1994. Infusion into the brain of an antisense oligonucleotide to the immediate early gene c-fos suppresses production of Fos and produces a behavioral effect. Neuroscience 63: 917-924.

Houpt, T.A., J.M. Philopena, T.C. Wessel, T.H. Joh, and G.P. Smith. 1994. Increased c-fos expression in nucleus of the solitary tract correlated with conditoned taste aversion to sucrose in rats. Neurosci. Lett. 172: 1-5.

Hughes, P. and M. Dragunow. 1995. Induction of immediate-early genes and the control of neurotransmitter-regulated gene expression within the nervous system. Pharmacol. Rev. 47: 133-178.

Hunter, J.C., V.L. Woodburn, C. Durieux, E.K.E. Pettersson, J.A. Poat, and J. Hughes. 1995. c-fos antisense oligodeoxynucleotide increases formalin-induced nociception and regulates preprodynorphin expression. Neuroscience 65: $485-492$

Inwin, K.B., A.D. Craig, V. Bracha, and J.R. Bloedel. 1992. Distribution of $c$-fos expression in brainstem neurons associated with conditioning and pseudoconditioning of the rabbit nictitating membrane reflex. Neurosci. Lett. 148: 71-75.

Kovary, K. and R. Bravo. 1991. Expression of different JUN and FOS proteins during the $\mathrm{G} 0$ to $\mathrm{G} 1$ transition in mouse fibroblasts: In-vitro and in-vivo associations. Mol. Cell. Biol. 11: 2451-2459.

Lamprecht, R. and Y. Dudai. 1996. Transient expression of $c$-fos in rat amygdala during training is required for encoding conditioned taste aversion memory. Learn. \& Mem. 3: 31-41.

Matthies, H. 1989. In search of cellular mechanisms of memory. Progr. Neurobiol. 32: 277-349.

McCabe, B.J. and G. Horn. 1993. Imprinting leads to elevated Fos-like immunoreactivity in the intermediate and medial hyperstriatum ventrale (IMHV) of the domestic chick. J. Physiol. 459: 160P.

Merchant, K.M. 1994. c-fos antisense oligonucleotide specifically attenuates haloperidol-induced increases in neurotensin/neuromedin $\mathrm{N}$ mRNA expression in rat dorsal striatum. Mol. Cell. Neurosci. 5: 336-344.

Möller, C., O. Bing, and M. Heilig. 1994. c-fos expression in the amygdala: In vivo antisense modulation and role in anxiety. Cell. Mol. Neurobiol. 14: 415-423.

Morgan, J.I. and T. Curran. 1989. Stimulus-transcription coupling in neurons: Role of cellular immediate early genes. Trends Neurosci. 12: 459-462.

Nikolaev, E., B. Kaminska, W. Tischmeyer, H. Matthies, and
L. Kaczmarek. 1992a. Induction of expression of genes encoding transcription factors in the rat brain elicited by behavioral training. Brain Res. Bull. 28: 479-484.

Nikolaev, E., T. Werka, and L. Kaczmarek. 1992b. c-fos protooncogene expression in rat brain after long-term training of two-way active avoidance reaction. Behav. Brain Res. 48: 91-94.

Paxinos, G. and C. Watson. 1986. The rat brain in stereotaxic coordinates, 2nd. ed. Academic Press, Sydney, Australia.

Paylor, R., R.S. Johnson, V. Papaioannou, B.M. Spiegelman, and J.M. Wehner. 1994. Behavioral assessment of c-fos mutant mice. Brain Res. 651: 275-282.

Pezzone, M.A., W.S. Lee, G.E. Hoffman, and B.S. Rabin. 1993. Induction of c-fos immunoreactivity in the rat forebrain by conditioned and unconditioned aversive stimuli. Brain Res. 597: 41-50.

Quercia, S. and S.L. Chang. 1996. Antisense oligodeoxynucleotide attenuates in vivo expression of c-fos in the paraventricular hypothalamic nucleus of the rat brain. Neurosci. Lett. 209: 89-92.

Robertson, G.S., W. Tetzlaff, A. Bedard, M. St.-Jean, and N. Wigle. 1995. c-fos mediates antipsychotic-induced neurotensin gene expression in the rodent striatum. Neuroscience 67: 325-344.

Sambucetti, L.C. and T. Curran. 1986. The fos protein complex is associated with DNA in isolated nuclei and binds to cellulose. Science 234: 1417-1419.

Scherczinger, C.A., A.A. Yates, and D.A. Knecht. 1992. Variables affecting antisense RNA inhibition of gene expression. Antisense strategies, Ann. N.Y. Acad. Sci. 660: $45-56$.

Schmidt, R., W. Brysch, S. Rother, and K.-H. Schlingensiepen. 1995. Inhibition of memory consolidation after active avoidance conditioning by antisense intervention with ependymin gene expression. J. Neurochem. 65: $1465-1471$.

Shih, C.-D., S.H.H. Chan, and J.Y.H. Chan. 1996. Participation of Fos protein at the nucleus tractus solitarius in inhibitory modulation of baroreceptor reflex response in the rat. Brain Res. 738: 39-47.

Sommer, W., B. Bjelke, D. Ganten, and K. Fuxe. 1993. Antisense oligonucleotide to c-fos induces ipsilateral rotational behaviour to $d$-amphetamine. NeuroReport 5: $277-280$.

Suzuki, S., P. Pilowsky, J. Minson, L. Arnolda, I.J. Llewellyn-Smith, and J. Chalmers. 1994. c-fos antisense in rostral ventral medulla reduces arterial blood pressure. Am. J. Physiol. 266: R 1418-1422.

Tischmeyer, W., L. Kaczmarek, M. Strauss, R. Jork, and H. Matthies. 1990. Accumulation of c-fos mRNA in rat 
hippocampus during acquisition of a brightness discrimination. Behav. Neural Biol. 54: 165-171.

Tischmeyer, W., R. Grimm, H. Schicknick, W. Brysch, and K.-H. Schlingensiepen. 1994. Sequence-specific impairment of learning by c-jun antisense oligonucleotides. NeuroReport 5: 1501-1504.

Wahlestedt, C. 1994. Antisense oligonucleotide strategies in neuropharmacology. Trends Pharmacol. Sci 15: 42-46.

Whitesell, L., D. Geselowitz, C. Chavany, B. Fahmy, S. Walbridge, J.R. Alger, and L.M. Neckers. 1993. Stability, clearance and disposition of intraventricularly administered oligodeoxynucleotides: Implications for therapeutic application within the central nervous system. Proc. Natl. Acad. Sci. 90: 4665-4669.

Wollnik, F., W. Brysch, E. Uhlmann, F. Gillardon, R. Bravo, M. Zimmermann, K.H. Schlingensiepen, and T. Herdegen. 1995. Block of c-Fos and JunB expression by antisense oligonucleotides inhibits light-induced phase shifts of the mammalian circadian clock. Eur. J. Neurosci. 7: 388-393.

Woolf, T.M., D.A. Melton, and G.B. Jennings. 1992.

Specificity of antisense oligonucleotides in vivo. Proc. Natl. Acad. Sci. 89: 7305-7309.

Received September 3, 1996; accepted in revised form January 9, 1997. 


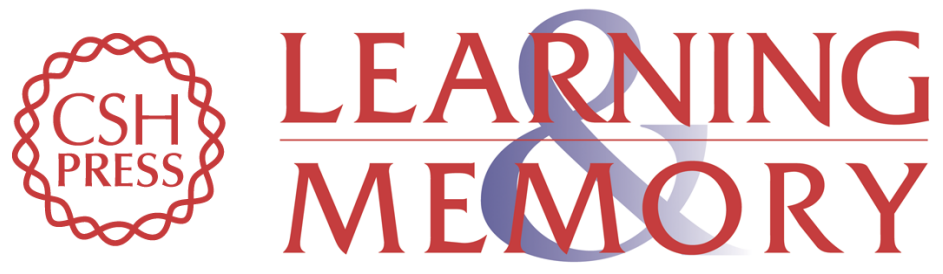

\section{Suppression of $\mathrm{c}$-fos induction in rat brain impairs retention of a brightness discrimination reaction.}

R Grimm, H Schicknick, I Riede, et al.

Learn. Mem. 1997, 3:

Access the most recent version at doi:10.1101//m.3.5.402

References This article cites 52 articles, 5 of which can be accessed free at: http://learnmem.cshlp.org/content/3/5/402.full.html\#ref-list-1

License

Email Alerting Receive free email alerts when new articles cite this article - sign up in the box at the Service top right corner of the article or click here. 